\title{
THE TECHNIQUE OF SURGICAL CORRECTION OF ANOMALIES OF THE PULMONARY VEINS IN A SERIES OF 25 CASES
}

\author{
BY \\ F. RISCH AND CHS. HAHN \\ From the Department of Thoracic Surgery, University of Leyden, Netherlands
}

(RECEIVED FOR PUBLICATION OCTOBER 27, 1958)

In this paper we intend to confine ourselves to the strictly surgical aspects of anomalies of the pulmonary veins in a series of 25 patients who were operated on in this clinic.

Anomalies of the pulmonary veins are very often associated with communications between the systemic and the pulmonary circulation, such as atrial septal defect, patent ductus arteriosus, or ventricular septal defect. An atrial septal defect existed in 23 of these 25 cases, and a ventricular septal defect in one ; only one patient showed an intact atrial septum (partial anomaly of the pulmonary veins). In none of these cases was a patent ductus arteriosus found. In two, the anomaly of the pulmonary veins was associated with coarctation of the aorta.
The various lesions are classified in Table $\mathrm{I}$.

TABLE I

CLASSIFICATION OF 25 CASES OF ANOMALIES OF PULMONARY VEINS TREATED SURGICALLY

Complete transposition of pulmonary veins $\quad$. $\quad . .24$ cases Partial transposition of pulmonary veins $\because 2 \quad \ldots \quad \ldots \quad 23$ cases 22 right-sided cases (all pulmonary veins in 7 , part of the veins in 15) One left-sided case

Complete Transposition of Pulmonary Veins

This is a rare anomaly. Guntheroth, Nadas, and Gross recently reported that only 159 cases have been described in the literature, usually as necropsy findings. Two cases were submitted to operation in this clinic. (Observations on all types of complete transposition of the pulmonary veins are presented in a paper by A. Dekker, of the Anatomical Institute, Leyden.)

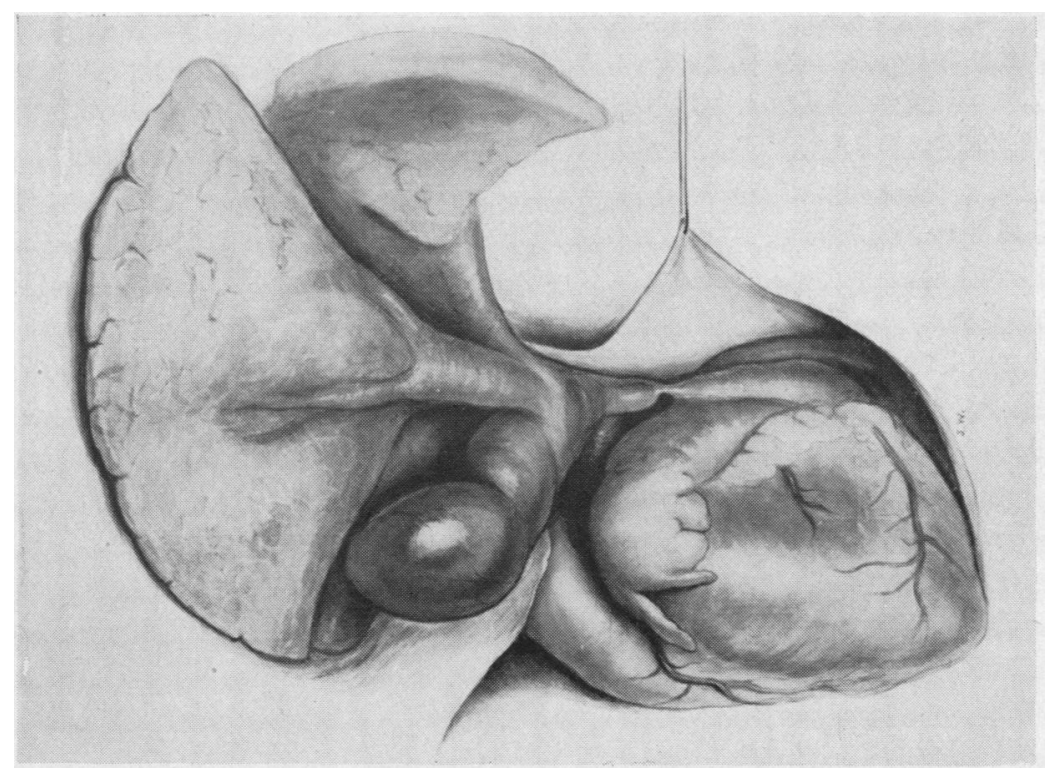

Fig. 1.-A drawing to show the large common trunk collecting the four pulmonary veins. Note the narrow anastomosis that in this case connects it with the coronary sinus. 


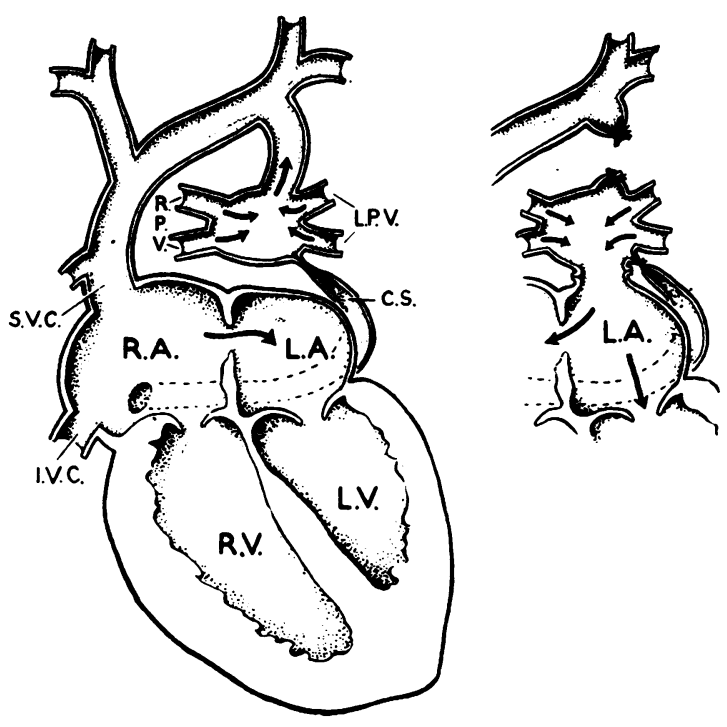

Fig. 2.-Pre-operative and post-operative condition schematically visualized. After correction the pulmonary veins drain into the left atrium. The atrial septal defect serves as a valve permitting the excess of pulmonary venous blood to escape to the right heart.

CASE 1.-A girl aged 5 underwent an operation in 1956. The four pulmonary veins terminated in what could be described as a common venous trunk (Fig. 1), communicating on the one hand with a large left superior vena cava draining into the right brachiocephalic venous trunk and, on the other hand, connected by a very narrow duct with the coronary sinus. At intracardiac palpation, the foramen ovale admitted the index finger.

This anomaly was corrected by making an anastomosis of $3.5 \mathrm{~cm}$. between the common trunk, collecting the four pulmonary veins, and the left atrium (Fig. 2). Marked pulmonary stasis with $50 \%$ desaturation of the peripheral arterial blood occurred during partial $(3 / 4)$ obstruction of the left superior vena cava that was required to make the anastomosis.

Once the anastomosis was open there was considerable dilatation of the left heart, but the pulmonary stasis disappeared. The left superior vena cava remained clamped for 20 minutes; when nothing happened, it was severed between two ligatures. Finally a ligature was placed on the narrow anastomosis connecting the common venous trunk with the coronary sinus.

In this case, the atrial septal defect was intentionally left open, although the operation was performed under hypothermia, so that it might act as a safety valve in case the left atrium should be incapable of coping with the additional blood flow.

Pulmonary stasis persisted throughout the first three post-operative weeks, after which the symptoms disappeared. The patient's present condition is excellent. The peripheral arterial saturation is clinically good, showing that such shunt as still exists has been reversed: it has become a left-right rather than a right-left shunt. Cardiac catheterization will soon be performed to determine whether or not the atrial septal defect must be closed.

CASE 2.-A boy aged $4 \frac{1}{2}$ years showed the same condition as the previous patient, different only in that there was no communication with the coronary sinus.

In this case, an anastomosis was made between the common venous trunk collecting the four pulmonary veins and the left atrium by means of a postero-
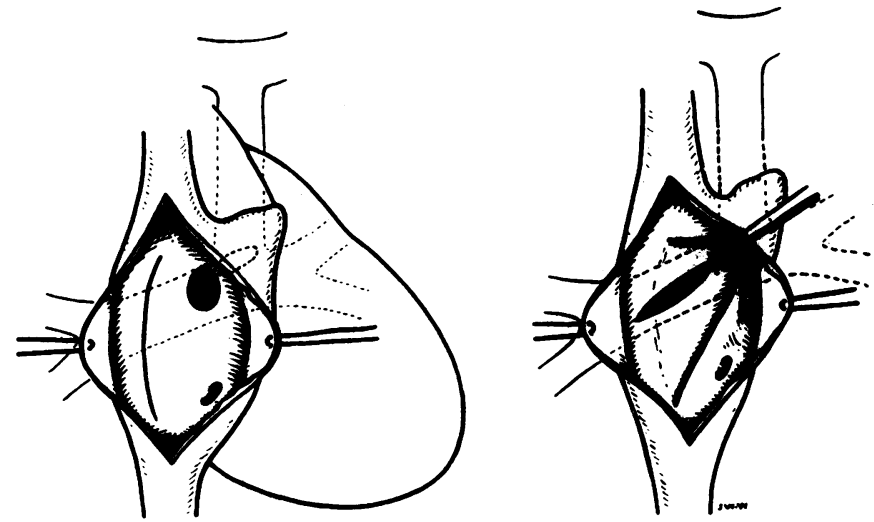

FIG. 3.-According to the technique of Cooley and Ochsner the atrial septum is separated, exposing the posterior wall of the left atrium in which a transverse incision is made to expose, in a way, the anterior wall of the trunk which drains the pulmonary veins. The incision in this common trunk is shown.

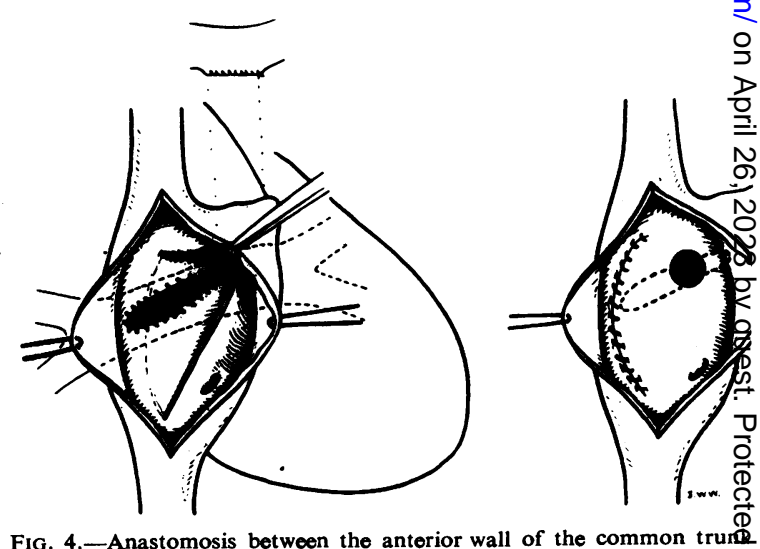

FIG. 4.-Anastomosis between the anterior wall of the common trui posterior wall of the left atrium. Re-insertion of the soptum $\underbrace{a}$ of the right auriculotomy. 
lateral thoracotomy, including the superior margin of the fifth rib. During the temporary ligation of the left superior vena cava, pulmonary venous pressure increased significantly, indicating insufficient drainage. In view of this development the incision was extended to become bilateral, involving a transverse sternotomy. Digital exploration of the cavities on the right revealed the existence of an atrial septal defect of the foramen ovale type.

Using extracorporeal circulation (Crafoord-Senning apparatus) and under cardiac arrest produced by potassium citrate, the condition was corrected by the technique described by Cooley and Ochsner (Figs. 3 and 4). The atrial septum was separated from the atrial wall, thus ensuring ample access to the left atrium and exposing the venous atrial anastomosis previously made. Afterwards an incision was made in the posterior wall of the left atrium and then in the anterior wall of the common trunk collecting the pulmonary veins. The margins of these two incisions were sutured together, thus ensuring ample communication between the pulmonary veins and the left atrium. Since this anastomosis extended slightly beyond the right atrial wall, the atrial septum was re-inserted at a site slightly more anterior. The small atrial septal defect was not closed, for the same reasons as those given in the previous case; its margins were freshened in the hope of subsequent spontaneous closure.

The left superior vena cava was then cut, and its stumps were sutured. Determination of pressure in the pulmonary veins and in the left atrium showed no gradient.

The perfusion lasted 30 minutes.

One feature in this case seems to be of great importance, viz., the greatly enlarged heart causing marked displacement of the two main bronchi in a superior direction; this explains some degree of bronchial compression, so that a tracheotomy had to be performed after the operation. Except for a small left-sided haemothorax with transient partial atelectasis of the left lung, the post-operative course was uneventful. At discharge from the clinic, the child was already in a condition considerably more favourable than before the operation. The cardiac volume had been reduced considerably.

\section{Partial Transposition of Pulmonary Veins}

Anomalous Termination of all Right PulMONARY VEINS.- Seven such cases were submitted to operation. Three of these were instances of pseudo anomalies concomitant with an atrial septal defect without a right lateral margin. In these cases, the right pulmonary veins showed too anterior a site of termination to the right of the normal insertion of the septum in the atrial wall.

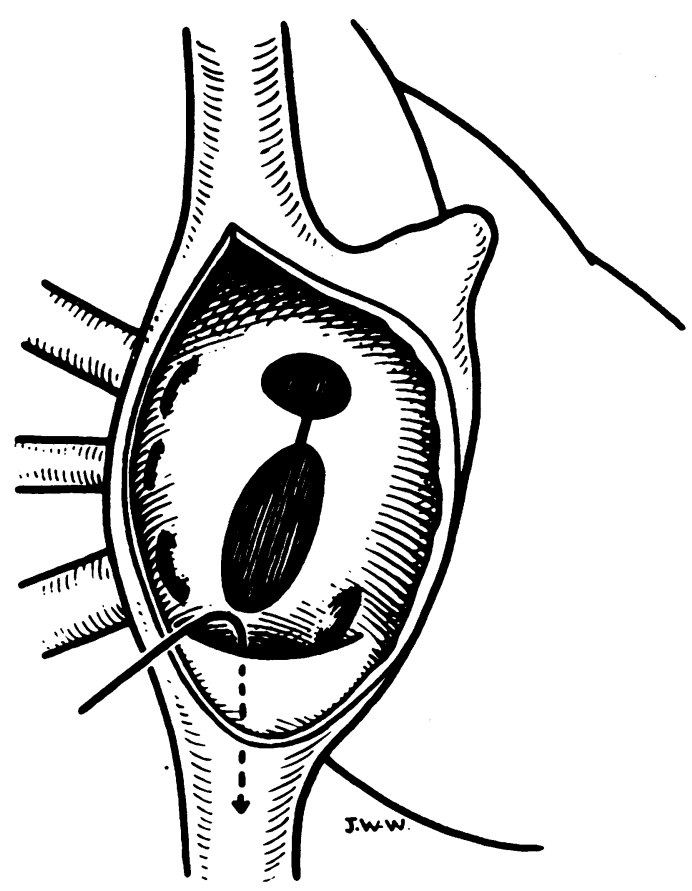

FIg. 5.-The right pulmonary veins, through the inedium of three lobar veins, drain into the right atrium. The two atrial septal defects are united. Note the large Eustachian valve (arrow)

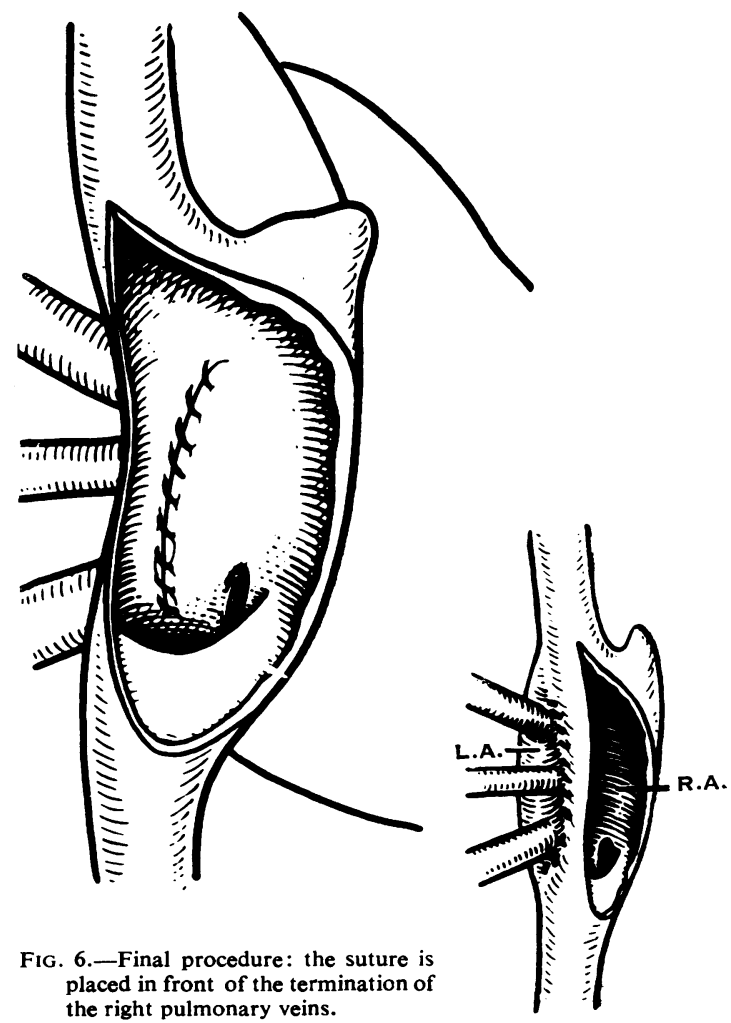


In such conditions it is sufficient to suture the median margin of the atrial septal defect on to the atrial wall in front of the site of termination of the veins; normal anatomy is thus restored.

Of these three cases, two progressed favourably, although considerable bronchial impairment necessitated a tracheotomy in one of them. The third patient died. This was a case of associated cardiovascular lesions, as coarctation of the aorta also existed. Since the latter malformation was not corrected during the same operation, it caused paraplegia and renal insufficiency after the mammary arteries had been severed. This case was a failure due to an error in surgical technique. (This patient was the first to be operated on under hypothermia in 1955.) At present, the coarctation in cases of this type is corrected first. In three cases the right pulmonary veins were implanted into the right auricle. In one case there was a patent foramen ovale in the form of a simple transverse crevice. From this crevice an incision was made in the atrial septum, and the median margin of the atrial septal defect thus obtained was sutured fast in front of the termination of the veins. In another case, two atrial septal defects (one a wide- open foramen ovale) were united by an incision, and the median margin of the one large atrial septal defect thus obtained was sutured fast in front of the termination of the pulmonary veins (Figs. 5 and 6). In the third case there was a condition resembling that in Case 2 but different in that the associated atrial septal defect had no inferior margin. This atrial septal defect was enlarged upwards, after which its median margin was sutured on to the wall of the atrium in front of the anomalous termination of three pulmonary veins.

CASE 8.-In the seventh case there was a curious anomaly, viz., one large pulmonary vein drained the blood of the right lung and terminated in the inferior vena cava at about $2 \mathrm{~cm}$. from the right atrium. On the other hand there was an atrial septal defect without a lateral margin (Fig. 7). In this patient, the right pulmonary artery was dissected out and clamped during the stage of correction. The right pulmonary vein was severed from the inferior vena cava and anastomosed with the left atrium, opposite the atrial septal defect. The latter was slightly enlarged downward during the intracardiac stage, after which its median margin was sutured in front of the anastomosis (Fig. 8).

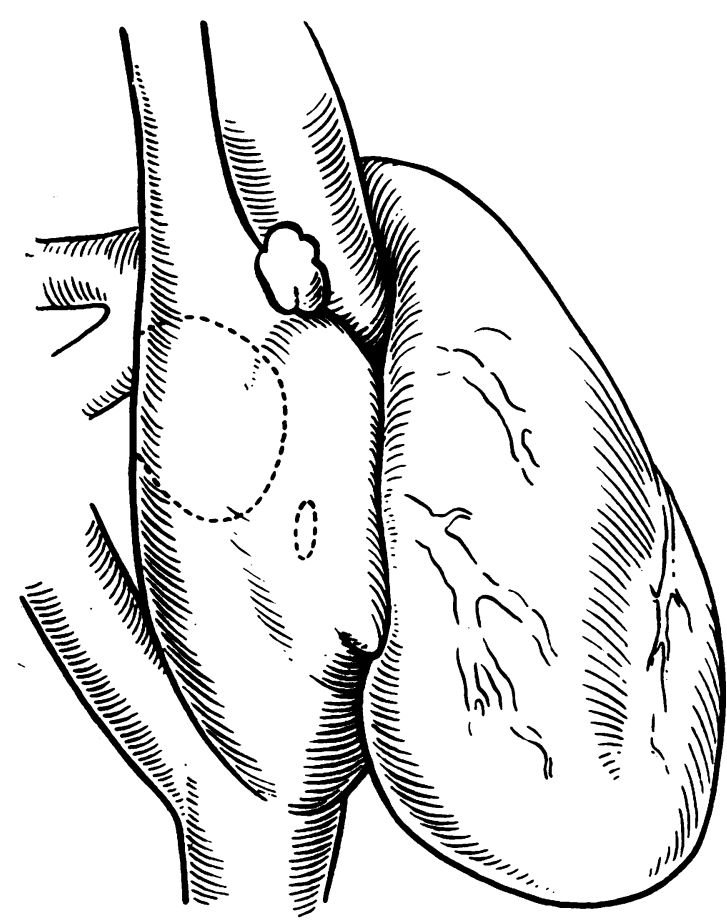

FIG. 7.-A large single right pulmonary vein draining into the inforior vena cava.

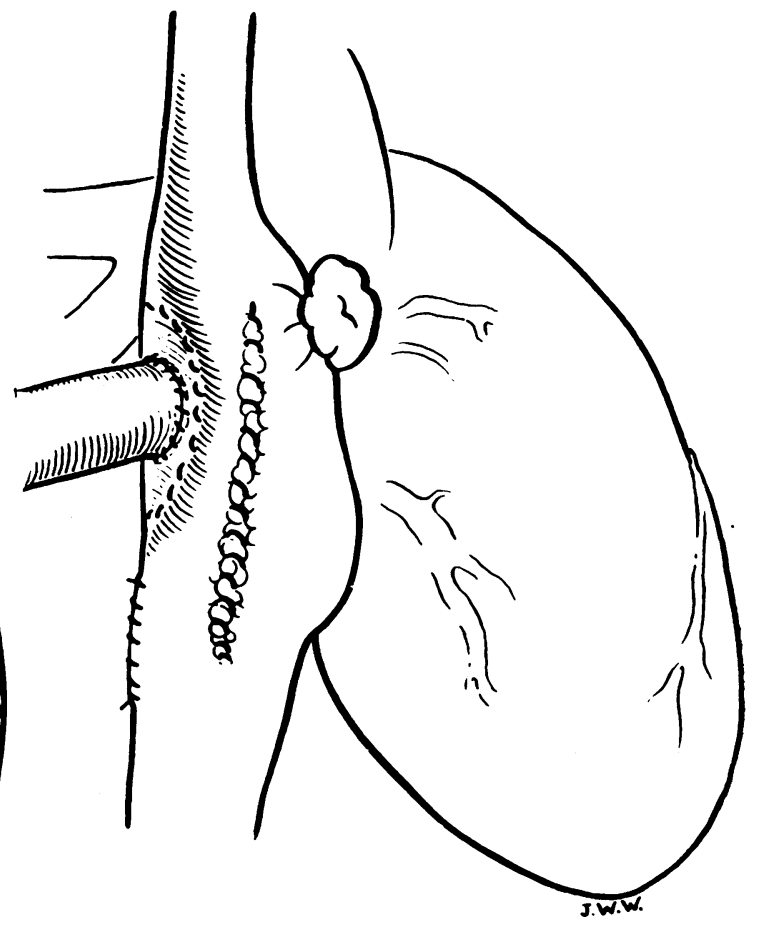

FIG. 8. - The right pulmonary vein is implanted into the left atrium. Closure of the atrial septal dofect (after downward onlargement) by suturing its modian margin in front of the anastomosis. 
These seven patients, like all those included in this series except one, underwent the operation under hypothermia. Only the last patient developed a complication, cardiac standstill during rewarming in a hot bath. Resuscitation was effective. The post-operative course in the last three cases was uneventful.

Anomalous Termination of Part of Right Pulmonary Veins.-This is the most frequently encountered form of all pulmonary venous transpositions. We have seen 15 cases. The condition was associated with an atrial septal defect in 13 and with a ventricular septal defect in one, one being without septal malformation.

Since repair of these lesions is largely a function of the localization of the septal defect, the 13 cases associated with atrial septal defect can be divided into four groups as follows:

(1) Venous transposition and central atrial septal defect .. 2 cases

(2) , , atrial septal defoct without

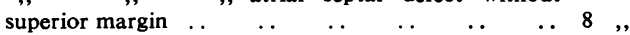

(3) Venous transposition and atrial septal defect without lateral margin $\quad . \quad$.

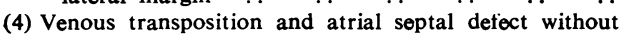

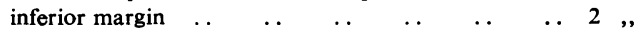

Partial Anomaly of Right Pulmonary Veins and Central Atrial Septal Defect.-The following cases are illustrative.

CASE 10.-In this patient the right superior pulmonary vein terminated in the right atrium, whereas the inferior pulmonary vein was normally implanted into the left atrium, opposite a central

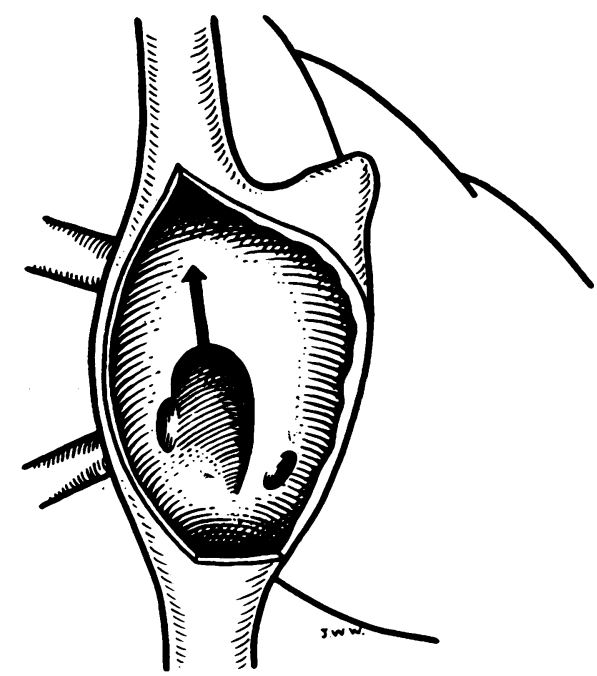

Fig. 9.-Contral atrial septal defect and patent foramen ovale. The right superior pulmonary vein drains into the right atrium. atrial septal defect. In addition, the foramen ovale was patent (Fig. 9). The atrial septal defect was enlarged upwards (arrow in Fig. 9) and the median margin of the orifice thus obtained was sutured fast in front of the termination of the right superior pulmonary vein. The foramen ovale was closed by means of a single stitch. The post-operative course was uneventful.

CASE 11.-This 11-month-old girl showed marked induration of the right superior lobe when the thorax was opened. The right superior lobar vein terminated in the azygos, while the middle and inferior lobar veins normally terminated in the right atrium. The central atrial septal defect was closed under hypothermia by the technique currently used at the clinic. Since transplantation of the right superior lobar vein could hardly be expected to be effective in view of the unsatisfactory condition of the superior lobe, a lobectomy was performed. Oxygenation of the blood was unsatisfactory during this intervention. Cerebral anoxia was prolonged, and the patient died a few hours after the operation.

Partial Anomaly of Right Pulmonary Veins and Atrial Septal Defect and without Superior Margin.-Eight cases have been submitted to operation; all showed an anomaly of the pulmonary veins. In this series, we distinguish three types.

(a) The first is pseudo anomalies of veins. In these cases, the pulmonary veins do not, properly speaking, drain into the right atrium but exactly opposite the median margin of the atrial septal defect, i.e., at the site at which the septum is normally implanted. In such it is sufficient to suture the median margin of the atrial septal defect on to the atrial wall in front of the anomalous terminations. We saw three cases of this type. In one, it was necessary to resort to the technique introduced by Schaepkens van Riempst and Brom (extension of the superior vena cava) in order to close the atrial septal defect effectively while enabling the pulmonary venous blood to return to the left atrium in the normal way (Figs. 10, 11, 12).

(b) The right pulmonary veins (and sometimes the middle lobar vein or veins) drain partly into the superior vena cava and partly into the right atrium at the site of termination of the superior vena cava in the right atrium. This condition was encountered once.

Case 15.-In this patient an extension of the superior vena cava at the expense of the atrial wall was impossible. Fortunately the left superior vena cava was more voluminous than the right. The latter could be ligated, the atrial septal defect being closed by suturing its median margin on to the right 


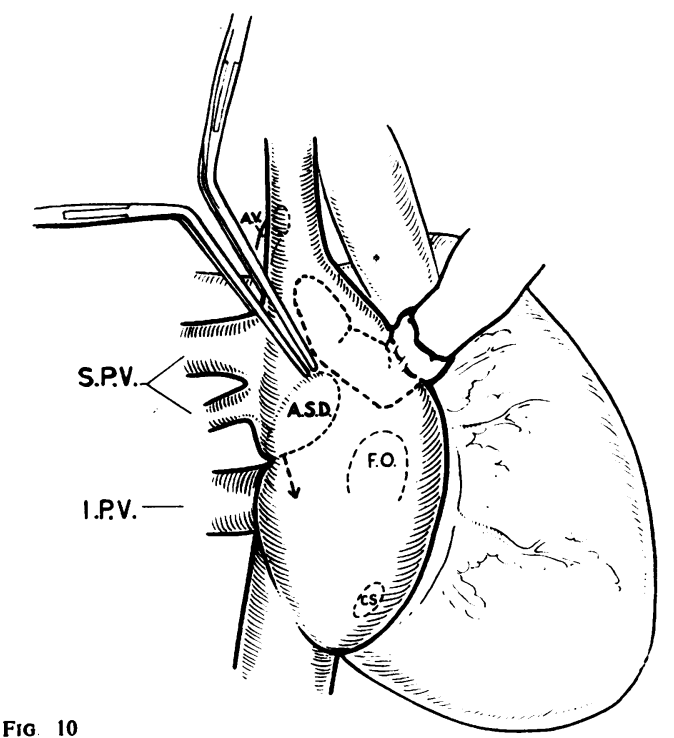

Fig. 10

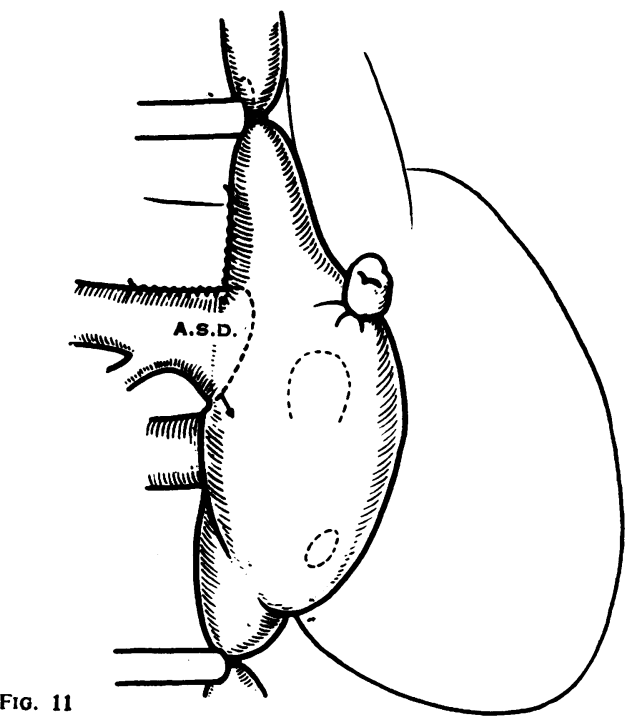

Fig. 11

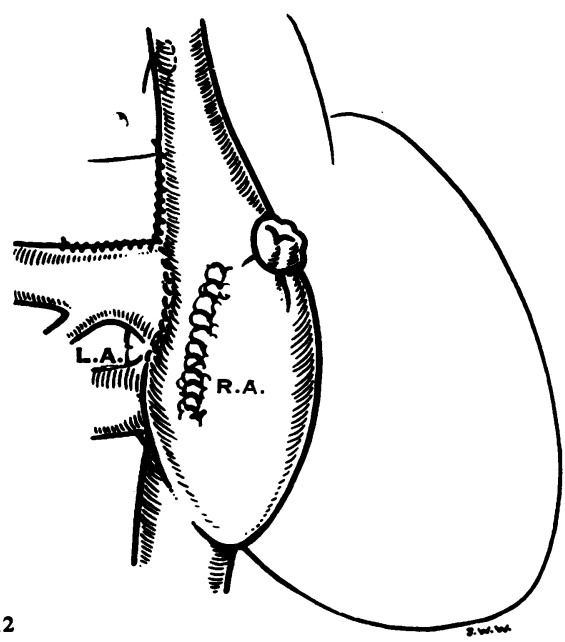

atrial wall, far in front, so that the anomalous pulmonary veins came to terminate in the left atrium.

(c) Part of the right pulmonary veins drain into the superior vena cava far from its termination in the right atrium ; the remainder of the pulmonary veins partly drain into the right atrium. In these cases the first procedure consists of transplantation of the vessel draining into the superior vena cava ; it is implanted into a subjacent pulmonary vein (Fig. 13), thus ensuring that all the right venous blood arrives in the auricles. After this it suffices to close the atrial septal defect (enlarging it if necessary) in front of the anomalous terminations; the normal anatomy is thus restored (Fig. 14). Extension of the superior vena cava by the method previously described may prove to be necessary. Four cases of this type were observed.

CASE 16.-In a woman aged 25 , the veins of the apical and the pectoral segments of the right lung formed a common trunk draining into the superior vena cava at the level of the termination of the azygos vein. The veins of the dorsal segment of the superior lobe and those of the middle lobe formed a common trunk draining into the right atrium near the termination of the superior vena cava, opposite a very small atrial septal defect. In addition, there was a large central atrial septal defect. In this case, the apicopectoral venous trunk was transplanted into the subjacent venous trunk. The high-seated defect was enlarged downward so that its median margin could be sutured on to the atrial wall in front of the termination of the trunk bearing the anastomosis.

CASE 17.- In a girl aged 13, one superior segmental vein drained into the superior vena cava, while the remainder of the right superior lobar vein terminated opposite the atrial septal defect, at the exact site at which the superior vena cava entered the right atrium. The right inferior pulmonary vein normally drained into the left atrium. Surgical repair was effected by transplantation of the superior segmental vein and downward enlargement of the atrial septal defect past the termination of the middle lobar vein. The median margin of the communication thus obtained was sutured in front of the middle lobar and the superior lobar veins.

CASE 18. - In a man aged 41 , the veins of the apical and dorsal segments of the right superior lobe drained into the superior vena cava near the termination of

Fig. 10.-Procedure according to the technique of Schaepkens van Riempst and Brom in which two clamps are placed at anglos on the atrial wall below the termination of the superior vena cava. A finger inside the caval vein controls its patency. A dimple shows the place of attachment of the lesser border of the defect to the atrial wall.

Fig 11-The cut sections are sutured, thus extending and displacing to the left the superior vena cava. The small arrow indicates the incision that makes it possible to onlarge the atrial septal dofect and to facilitate mobilization of the septum.

FIG. 12.-Extension of the superior vena cava facilitates simple and effective closure of the atrial soptal defect, whilo restoring the right pulmonary flow to normal. 


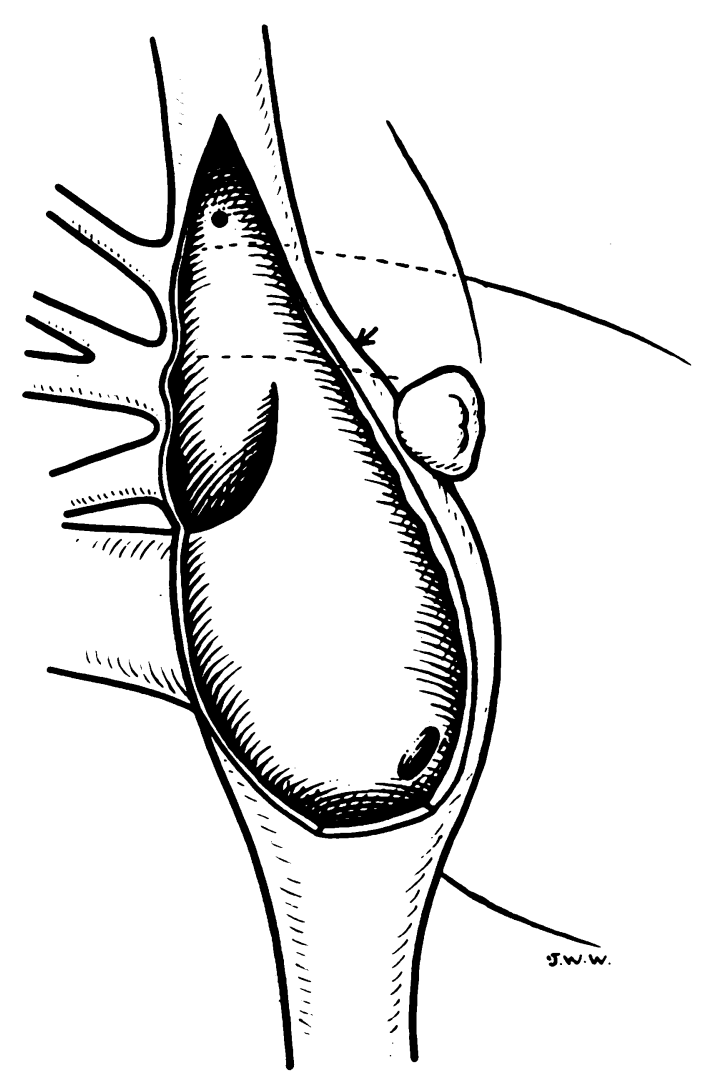

FIG. 13.- In this drawing the superior vena cava has been incised to demonstrate the relations between the various anomalous veins.

the azygos vein. The remainder of the superior lobar vein terminated opposite the atrial septal defect, but at a certain distance from the termination of the superior vena cava in the right atrium. This made it possible to use our method of extending the superior vena cava after transplantation of the superior segmental veins into the trunk of the superior lobar vein. The superior vein, thus reconstructed, was much nearer the atrial septal defect, the median margin of which could be sutured on to the atrial wall without causing stenosis of the superior vena cava. In this case the foramen ovale was also patent ; it was closed by crosswise suture.

CASE 19.--In this boy aged 14, a segmental vein of the right superior lobe drained into the superior vena cava, while the other two segmental veins of the same lobe formed a short common trunk draining into the right atrium. The middle lobe vein also drained into the right atrium, opposite the lower part of the atrial septal defect. After transplantation of the superior segmental vein into the common trunk of the two subjacent veins (see Fig. 13), the superior vena cava was extended. This was followed by auri-

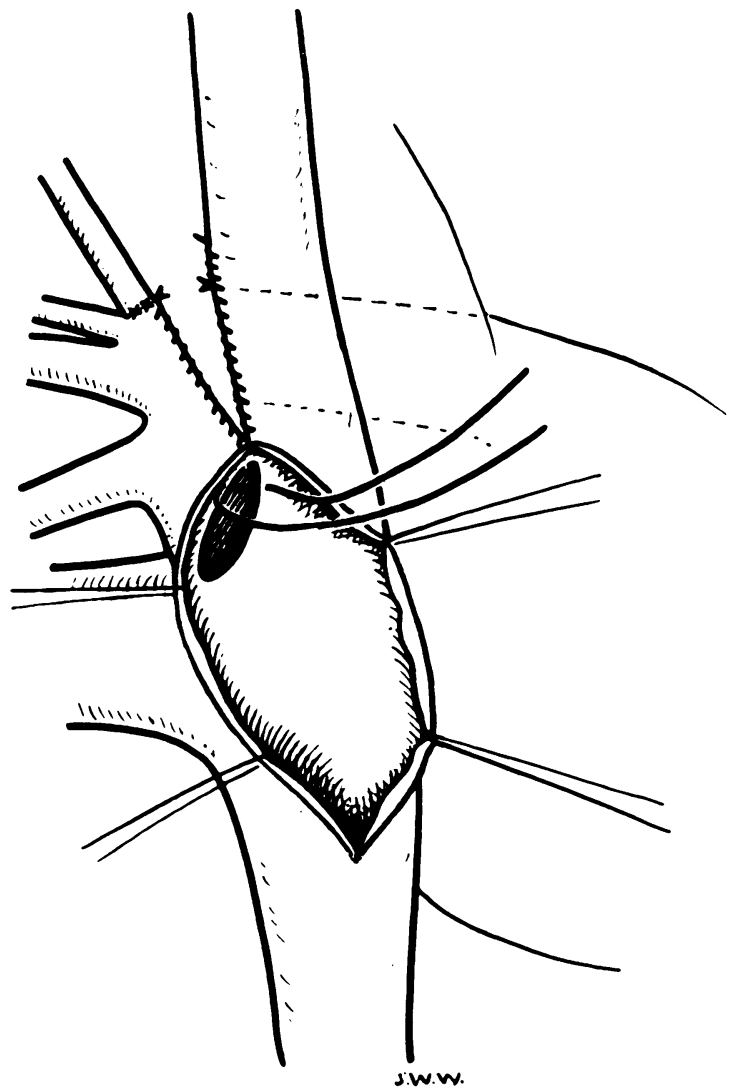

FIG. 14.-After transplantation of veins and extension of the superior vena cava, the atrial septal defect is sutured in front of the anomalous termination of the superior pulmonary veins.

culotomy and closure of the atrial septal defect in front of the anomalous termination (see Fig. 14).

Partial Anomaly of Right Pulmonary Veins and Atrial Septal Defect without Lateral Margin.This condition was seen once.

CASE 20.- In a 35-year-old man, the veins of the upper and middle right lobes drained into the right atrium, slightly above the upper margin of an atrial septal defect without a right lateral margin. The superior vena cava defect was extended, after which the defect was enlarged downward so that its median margin could be sutured fast in front of the anomalous terminations.

Partial Anomaly of Right Pulmonary Veins and Atrial Septal Defect without Inferior Margin.This condition was seen twice.

CASE 21.-In this patient there was a pseudo anomaly of the veins. The septum was sutured in front of the termination of the right inferior pulmonary vein. 


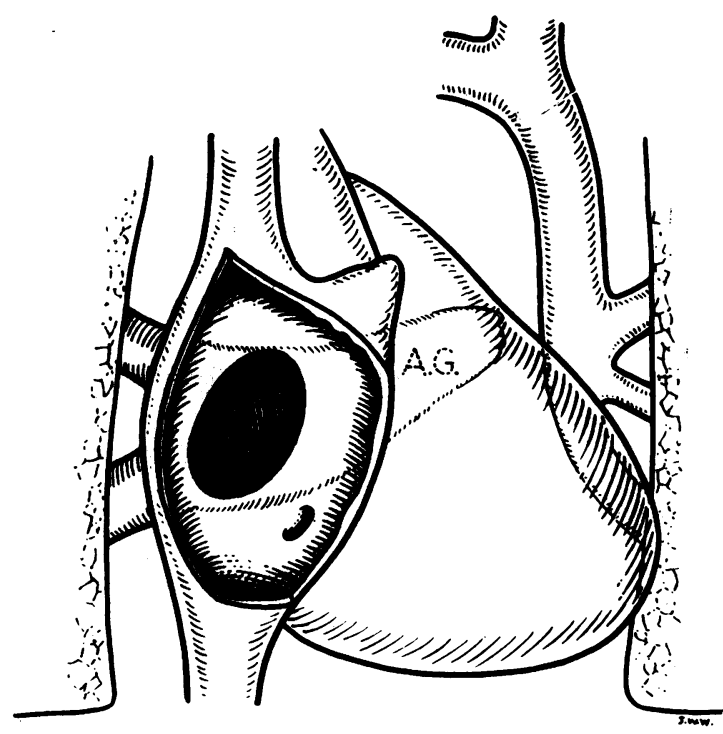

FIG. 15.-One large left superior vena cava drains the entire volume of blood from the left lung. The dotted line shows the contours of the left atrium that will be used for an anastomosis between the vein and the atrium. The atrial septal defect is large.

CASE 22.-The right inferior pulmonary vein in this case drained openly into the right atrium. The atrial septal defect was enlarged upward so that its median margin could be sutured in front of the inferior pulmonary vein.

Partial Anomaly of Right Pulmonary Veins and Ventricular Septal Defect.-An operation was performed in one case of this type.

CASE 23.-In a 10-year-old girl, the vein of the right upper lobe drained into the superior vena cava, about $2.5 \mathrm{~cm}$. from its termination in the right atrium. In addition there was a ventricular septal defect in the membranous septum and slight insufficiency of the tricuspid valve. In this case the defect was closed with the aid of extracorporeal circulation (perfusion lasted 14 minutes) by a simple suture. During the time required to perform this operation, ventricular fibrillation occurred (no provoked cardiac standstill). After closing the defect, a partial bypass was maintained for 10 minutes, and, once the myocardium was well oxygenated, defibrillation was effected by means of a single electrical shock.

The vein of the right upper lobe was reimplanted directly into the left atrium.

Absence of any shunt was demonstrated by determination of oxygen saturation in blood samples obtained from the superior vena cava, the right atrium, and the pulmonary artery. The post-operative course was uneventful.

Partial Anomaly of Right Pulmonary Veins without Septal Defect.-One case was observed.

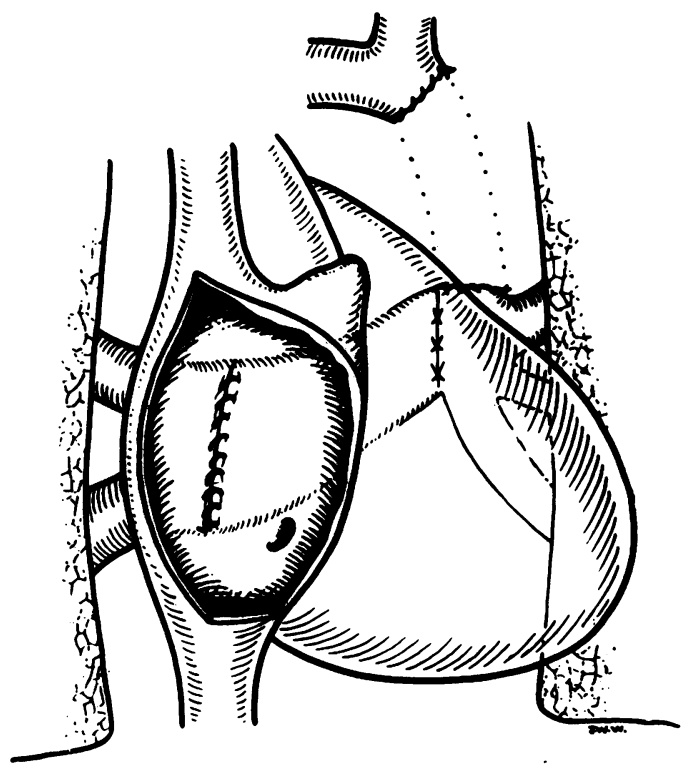

FIG. 16.-Anastomosis between the vein and the left atrium. Severance of the left superior vena cava and suture of the two stumps. Closure of the defect.

CASE 24.-In a 16-year-old youth, the apicodorsal, pectoral, and middle lobar veins drained into the right atrium. In addition, the patient suffered from coarctation of the aorta. The coarctation was resected through a left-sided thoracotomy, and aortic continuity was restored by end-to-end anastomosis. A transverse sternotomy was then used to turn the unilateral incision into a bilateral thoracotomy. The circulation was interrupted, and an atrial septal defect was created by incising the septum near its insertion in the atrial wall, after separating the superior vena cava from the apicodorsal and pectoral veins by means of a series of stitches according to the technique described by Bailey. The atrial septum was then re-inserted in front of the venous terminations. During the post-operative period a tracheotomy had to be performed because of bronchial impairment and blood-tinged sputum. Stasis of the centre right lung occurred and lasted about two weeks. A rightsided haemothorax was punctured. The late results were good, but we will have to investigate his lung function.

Anomalous Termination of Left Pulmonary Veins.-Only one case of this type was seen.

CASE 25.-In a woman aged 27 , the entire volume of left pulmonary blood was drained by a large left superior vena cava connected with the right brachiocephalic venous trunk (Fig. 15).

There was a large central atrial septal defect. An anastomosis of a diameter equal to that of the atrium was made between the left atrium and the left superior vena cava, facing the termination of the left 
pulmonary veins. The vena cava was then resected, and its stumps were sutured (Fig. 16). This was followed by a right-sided auriculotomy and closure of the defect by the ustial technique.

\section{Results}

The following results were obtained in all surgical cases of transposition of pulmonary veins in this series.

Treatment was successful in both cases (one tracheotomy) of complete transposition.

In seven cases of partial transposition affecting all right pulmonary veins, treatment was successful in six. One death occurred, due to an error in surgical technique (coarctation was not controlled during the same stage of the operation). During the operation the heart stopped in one case, but the patient was successfully resuscitated, and in another ventricular fibrillation followed removal of the clamps. Defibrillation was successful. Post-operatively in one case respiratory disturbances (haemothorax, partial pulmonary atelectasis) were controlled by tracheotomy.

In 15 cases of partial transposition involving only part of the pulmonary veins, two patients died.

During operation complications were encountered in three cases, one of ventricular fibrillation after removal of the clamps, but defibrillation was effected without difficulty. One patient, an 11month-old child, suffered cerebral anoxia during supplemental lobectomy and died. We had one case of coronary air embolism without ill effects.

Post-operative complications included one case of bilateral haemothorax with pulmonary and cardiac compression (fatal issue); one case of mild cardiac failure ; one case of bronchial impairment treated by tracheotomy, after which progress was uneventful ; one case of paroxysmal tachycardia, which disappeared after four days' treatment with digitalin ; one case of pyrexia of obscure origin. One case of suspected thrombosis of an anastomosis following transplantation, and one of pulmonary stasis in the lung segment drained by the transplanted vessels, merit certain comments. Initially, pressure was not determined after transplantation or implantation of veins. (These two cases are included in this first series of patients.) At present, the pressures in the transplanted veins and in the left atrium are determined after each anastomosis of veins. This is done by means of a cannula introduced either into the left atrium if a bilateral thoracotomy was made or, through a short incision, into a pulmonary vein if the thoracotomy was confined to the right side. If there is a gradient in pressure, then the anastomosis must be remade or a lung resection done. Since we have made a routine of these checks, no case of post-operative pulmonary stasis has been seen in which the classical symptoms resemble those of pulmonary arterial infarction, viz., bloodtinged sputum and opacification of the parenchyma.

We have adopted the following rules to be observed in all cases requiring an anastomosis of veins :

(1) The anastomosis should be as wide as possible.

(2) Pressure determinations should be made after completion of the anastomosis by means of a cannula because determination of venous pressures is not sufficiently exact if made with a needle (insufficiently accurate detection of slight differences).

(3) Very exact supervision of the clinical condition (blood-tinged sputum) and the radiological aspects (parenchymal opacification) after operation is necessary.

One case of total anomaly of the left pulmonary veins was operated on, and during operation ventricular fibrillation due to coronary air embolism occurred but was successfully defibrillated. Late results were excellent.

\section{ConCLUSIONS}

Anomalies of the pulmonary veins, whether total or partial, are nearly always associated with septal malformations, viz., atrial or ventricular septal defects.

Complete transpositions are amenable to effective repair without exclusion of the heart. It is desirable to have a heart-lung apparatus ready and at hand in case the Cooley-Ochsner procedure should become necessary. In such we believe that it is advisable not to close the atrial septum completely until more information has been gathered : the persistence of a communication between the left and the right atrium permits of progressive haemodynamics adaptation while avoiding an excess of blood in the left heart. On the other hand, where the heart volume has caused deformity of the main bronchi, we consider it advisable to supplement the intervention by a subsequent tracheotomy to control such pulmonary complications as may result.

As regards partial transpositions affecting the veins of the right lung (all of them or only some of them), we believe that an operation under 
hypothermia is the method of choice whenever atrial septal defect is present. This permits repair of the two lesions. In anomalies of the right pulmonary veins not associated with an atrial septal defect, hypothermia is likewise indicated as it allows separation and re-insertion of the septum in front of the anomalous terminations. If a ventricular septal defect is associated, however, then extracorporeal circulation is indispensable, and we have used the Crafoord-Senning apparatus.
Anomalies of the left pulmonary veins, when associated with atrial septal defect, are also amenable to correction under hypothermia.

We are indebted to Professor Brom for allowing us to study the patients treated in his clinic.

Acknowledgments are due to Miss J. Whittaker for the drawings. These constitute her valuable contribution to this paper.

REFERENCE

Cooley, D. A., and Ochsner, A. Jr. (1957). Surgery, 42, 1014. 